\title{
DEVELOPMENTS IN BEAM-BASED ALIGNMENT AND STEERING OF THE NEXT LINEAR COLLIDER MAIN LINAC
}

\author{
P. Tenenbaum, L. Hendrickson, T.O. Raubenheimer, SLAC *
}

\begin{abstract}
The Next Linear Collider main linacs are $13 \mathrm{~km}$ linear accelerators which each contain approximately 750 hybrid iron/permanent-magnet quadrupoles in a FODO array. The small amount of vertical emittance dilution permitted in the main linacs implies a tight tolerance on the RMS distance between the beam and the centers of the quads. We describe two methods for measuring the offsets between the quads and their integrated beam position monitors, and three algorithms for steering the main linac to minimize the emittance dilution. Simulation studies of the alignment and steering algorithms are presented.
\end{abstract}

\section{INTRODUCTION}

The principal element of the Next Linear Collider (NLC) which achieves the desired center-of-mass energy (up to 1 $\mathrm{TeV}$ ) is the main linacs. Each of the two main linacs is approximately $13 \mathrm{~km}$ long and contains over 10,000 X-band $(11.424 \mathrm{GHz})$ accelerator structures, each $0.9 \mathrm{~m}$ long, interleaved with approximately 750 quadrupoles. Each of the linacs must accelerate the beams from $8 \mathrm{GeV}$ to $500 \mathrm{GeV}$, while at the same time increasing the normalized vertical emittance of each beam by no more than $8 \times 10^{-9} \mathrm{~m} . \mathrm{rad}$.

For a high-frequency linear accelerator, the most significant cause of emittance dilution is the long-range and shortrange beam-break-up instability (BBU), which are caused by the excitation of dipole modes in the RF structures. The long-range BBU instability is addressed through direct reduction of the wakefield: this is accomplished through a combination of light damping of the dipole modes and detuning the dipole mode frequencies in an 83-cell RF structure [1]. The impact of the short-range BBU is minimized by introduction of a longitudinally-correlated energy spread, which causes the lattice chromaticity and the effect of the structure wakefields to cancel [2]; in addition, the $\mathrm{RF}$ structures are mounted on remote-controlled translation stages which are moved until the dipole mode power, measured directly in the damping manifolds of the structures, is minimized [3]. The combination of a correlated energy spread of approximately $0.6 \%$ per $\sigma_{z}$ and RF structure positioning resolution at the micrometer level can reduce the contribution of short-range BBU to the normalized emittance to well below $1 \times 10^{-9} \mathrm{~m}$.rad.

The introduction of the energy spread for the damping of BBU makes the tolerance on quadrupole alignment severe: an RMS beam-to-quad misalignment of $2.5 \mu \mathrm{m}$ will add $8 \times 10^{-9} \mathrm{~m} . \mathrm{rad}$ to the normalized emittance. Each

\footnotetext{
* Work Supported by the U.S. Department of Energy, Contract DEAC03-76SF00515.
}

quadrupole contains a beam position monitor (BPM) with a single-pulse resolution of $0.3 \mu \mathrm{m}$, and is mounted on a remote-controlled translation stage with $0.05 \mu \mathrm{m}$ step size. In principle, these tools would permit the magnets to be moved such that the RMS beam-to-quad offset is small. In practice, the reduction of the beam-to-quad RMS offset is limited by two factors: the offsets between the BPM electrical centers and the quadrupole magnetic centers, which are not known ab initio and must be measured (the alignment problem); and the fact that the algorithm which moves the quads to achieve a small RMS beam-to-quad offset must not drive the translation stages out of range and must obey other "real-world" limitations (the steering problem).

\section{SOLVING THE ALIGNMENT PROBLEM}

The time-honored solution for measuring the BPM-toquad offsets in a beamline is to vary each quadrupole's focusing strength in turn and measure the resulting deflections in downstream BPMs. This permits the beamto-quad offset to be directly measured: $\Delta x_{\mathrm{BPM}}=$ $R_{12}^{\text {quad } \rightarrow \text { BPM }} \Delta K_{\text {quad }} x_{\text {beam-to-quad. }}$ The BPM-to-quad offset is the difference of the BPM reading and the beamto-quad offset. In the Final Focus Test Beam (FFTB) at SLAC, statistical resolutions of the beam-to-quad offset of less than $1 \mu \mathrm{m}$ were achieved with modest averaging [4], and analytic estimates have indicated that comparable resolutions can easily be achieved in the NLC main linac [5].

The main limitation of the quadrupole-variation technique appears to be the systematic fitting error which occurs when the quadrupole's magnetic center changes its position as the quad strength is varied. To lowest order, the resulting error is proportional to the magnitude of the center motion and inversely proportional to the fractional change in quad strength [5]. Thus, if the center of the quad moves by $1 \mu \mathrm{m}$ when its strength is varied by $20 \%$, the quad-centering fit will have a $5 \mu \mathrm{m}$ systematic error.

One approach that will minimize the systematic error is to reduce the quad strength to zero, or as close as will be permitted by the remnant field of the pole pieces. In this case, known as ballistic alignment, the systematic error above is negligible [6].

The present design calls for the NLC main linac quadrupoles to be energized by blocks of permanent magnet (PM) material rather than electrical windings [7]. The NLC quads will attain some degree of strength adjustment through use of PM elements which are physically rotated or otherwise moved, but the degree of adjustability will probably not exceed $20 \%$. Therefore, the NLC main linac cannot use the ballistic alignment technique. 


\subsection{Dispersion Free Steering}

Dispersion Free Steering (DFS) is a technique for directly measuring and correcting anomalous dispersion in a beam transport line [8]. In DFS, the beam energy (or in the case of a linac, energy gain) is varied and the resulting change in the beam trajectory is measured. The change in orbit with energy - dispersion - is used to calculate corrector settings that will steer the beam to an orbit where the dispersion is minimized. DFS is a technique for correcting emittance dilution due to anomalous dispersion that relies only upon BPM resolution, and does not require direct measurement of BPM offsets. Its principal disadvantage is that it does not locally correct emittance dilution, as standard beam-based alignment does, and therefore is a less local, less stable correction. Also, like all alignment algorithms, it is assumed in DFS that the resulting correction to the orbit also correlates to an improved emittance, even though the emittance is not directly used as an input by the algorithm.

DFS has been used successfully in the SLAC linac, in the form of "two beam dispersion free steering:" in this application, the difference orbit between the electron and positron bunches in the linac was minimized, rather than the difference between two electron beam orbits with different energy profiles [9]. DFS was also used in the LEP ring to achieve record performance [10].

One factor which complicates the application of DFS to the NLC linacs is RF steering: transverse deflections in accelerating structures which might vary as the amplitude or phase of the RF power is changed. This would cause a systematic error in the measured dispersion similar to the one caused by the quadrupole center shift discussed above. This can be ameliorated by dividing the linac into subsections for alignment, and then varying the energy gain upstream of the region which is being aligned but not within the region. Any steering from RF variation will thus constitute a change in the initial condition of the beam, which can be removed by fitting or by steering, rather than an anomalous dispersion.

The DFS algorithm described above has been simulated for the NLC linac using the program LIAR [11]. The linac was divided into 25 DFS regions, which were aligned sequentially. Realistic RF structure misalignments and instrumentation performance were assumed in the simulation. Assuming that the BPMs in the quadrupoles achieve a resolution of $1 \mu \mathrm{m}$, DFS can reduce the emittance dilution in the NLC main linac to approximately $1.6 \times 10^{-8}$ m.rad. Although this is a small amount of emittance dilution, it remains twice as large as the budgeted dilution from all sources in the linac. Improvement of the BPM resolution to $0.3 \mu \mathrm{m}$ reduced the emittance dilution to $0.4 \times 10^{-8}$ m.rad, which is only half the total budgeted dilution.

\subsection{Emittance Bumps}

An additional technique for reducing emittance dilution in a linac is to introduce closed orbit oscillations which can generate dispersion. The amplitude of these oscillations is tuned to minimize the beam size on a properly located profile monitor. This technique was used with great success throughout the lifetime of the SLC [12]. In the NLC main linac true dispersion bumps can be introduced because the RF structure girder translation stages can be used to realign the girders to the beam within the region of the oscillation.

A simulation study of the NLC main linac was performed in which first DFS and then emittance bumps were applied. A total of 14 emittance bumps were employed: one per betatron phase main linac injection, extraction, and 5 locations in-between. Each bump was tuned on an appropriate profile monitor location downstream in a manner quite analogous to the tuning technique employed in the SLC. When $1 \mu \mathrm{m}$ BPM resolution was assumed in the DFS operation, the combination of DFS+bumps limited emittance dilution to $0.6 \times 10^{-8} \mathrm{~m} . \mathrm{rad}$; when $0.3 \mu \mathrm{m} \mathrm{BPM}$ resolution was used, the emittance dilution was limited to $0.16 \times 10^{-8} \mathrm{~m} . \mathrm{rad}$. Either of these would be a satisfactory solution for the NLC main linac.

\section{STEERING THE MAIN LINAC}

The procedures outlined above will allow determination of a beam trajectory which results in acceptable emittance at the end of the linac. In a static environment, this procedure could be performed once and the linac emittance would remain acceptable for all time. In reality, slow drifts in the positions of beamline elements will slowly drive the beamline away from its desired condition. Sources of such drifts include diffusive motion (often called "ATL motion" [13]), and systematic motion, such as differential settling of the tunnel [14]. In order to maintain the main linac performance, it will be necessary to periodically re-steer the linac to recover the "gold orbit" which corresponds to the desired performance.

\section{$3.1 \quad$ Steering Feedback}

The SLAC linac uses a series of feedback loops to automatically maintain the beam orbit on a subset of BPMs by adjusting a small number of steering dipoles [15]. The NLC main linac will utilize a similar system, but with a more sophisticated configuration which is expected to provide much better performance [16].

Figure 1 shows the evolution of the emittance at the end of the NLC main linac under the influence of diffusive ground motion; an "ATL" coefficient of $5 \times 10^{-7} \mu \mathrm{m}^{2} / \mathrm{m} / \mathrm{sec}$ is assumed; this value is based on recent measurements of such motion in the SLAC accelerator housing [17]. In the absence of steering feedback, the beam quality is degraded unacceptably within a few minutes. A set of 9 feedback loops are sufficient to maintain the desired emittance for several hours. After approximately 8 hours, the orbit between feedback regions has changed so much that even with the feedback loops unacceptable emittance dilution is observed. 


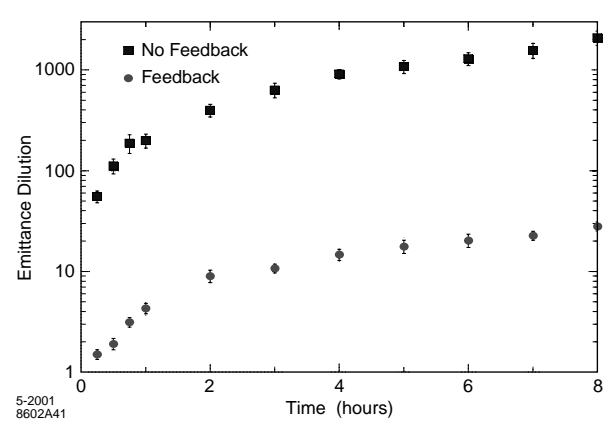

Figure 1: Emittance vs time with ATL motion, with (squares) and without (circles) linac steering feedback.

\subsection{Mover Steering}

The procedures described in Section 2 are too invasive to be frequently invoked. Consequently, some intermediate solution is required for stabilization of the main linac emittance over time scales which are too long for the steering feedback but too short to permit use of DFS.

One approach is to frequently re-steer the main linac, by moving quads and RF structure girders via their translation stages, to recover the "gold orbit." In principle, if the transport matrices of the linac are known with sufficient accuracy, the entire linac can be steered in one iteration, which would require a single inversion of a very large steering matrix. This is not desirable for several reasons. First, the RF structure wakefields complicate the beam transport dynamics: over short stretches of linac the transport is simple to model but the effective $R$-matrix elements between points separated by kilometers are difficult to determine. Second, The problem must be constrained in such a way as to generate solutions for which all translation stages are within their range limits.

Three different algorithms for linac steering with magnet movers have been explored [18]. Each algorithm divides the NLC linac into subsections, and steers the subsections sequentially from upstream to downstream. In the first algorithm, employed in the NLC design study [19], the first and last quad in a subsection are held fixed and the intervening quads are moved such as to simultaneously minimize the RMS BPM difference from gold and the RMS magnet mover motion; steering correctors are used to link the beam trajectories between subsections. This algorithm was found to have several disadvantages. First, the algorithm requires the addition of steering dipoles at the intersections between subsections to match the steering between subsections. Second, the emittance dilution from this algorithm tends to be somewhat larger than desired. Third, the algorithm is unacceptably sensitive to magnet mover step size: a step size of $300 \mathrm{~nm}$ produces much more emittance dilution than a step size of $50 \mathrm{~nm}$.

A refinement of the algorithm above employs first the ZDR algorithm on a given subsection, and then MICADO, before advancing to the next subsection [20]. This addition eliminated the sensitivity to mover step size, but otherwise shares the deficiencies of the ZDR algorithm.

A third algorithm was designed which is also similar to the ZDR procedure; in this case, no correctors are used. Instead, after a subsection is aligned the procedure advances by only half a subsection. Thus, a quad which is an endpoint quad for one subsection is a middle quad in the next one. This algorithm, though slower than the ZDR one, has a smaller equilibrium emittance, no sensitivity to mover step size, and requires no additional steering dipoles in the linac.

\section{CONCLUSIONS}

We have investigated a variety of techniques for determining and maintaining a high-performance orbit in the NLC main linac. The determination of the orbit will, if possible, use quadrupole variation to measure quad-to-BPM offsets; if this proves untenable, a combination of DFS and bumps will be used. The performance will be preserved by a combination of linac steering feedback loops and periodic resteering of the orbit via quadrupole magnet movers. There appear to be no fundamental obstacles to attaining and preserving the desired emittance at the end of the main linac.

\section{REFERENCES}

[1] R. Jones et al, these proceedings.

[2] V.E. Balakin, A.V. Novokhatsky, V.P. Smirnov, Proceedings HEACC-83, 119 (1983).

[3] C. Adolphsen et al, Proceedings PAC-99, 3477 (1999).

[4] P. Tenenbaum et al, Proceedings IWAA-95, 393 (1995).

[5] P. Tenenbaum and T.O. Raubenheimer, PRST-AB 3:052801 (2000).

[6] T.O. Raubenheimer, D. Schulte, Proceedings PAC-99, 3441 (1999).

[7] J. Volk et al, "Adjustable Permanent Magnet Quadrupoles for the Next Linear Collider," these proceedings.

[8] T.O. Raubenheimer, R.D. Ruth, NIM A302:191 (1991).

[9] P. Raimondi et al, "Recent Luminosity Improvements at the SLC," Proceedings HEACC-98 (1998).

[10] R. Assmann et al, PRST-AB 3:121001 (2000).

[11] R. Assmann et al, SLAC-AP-103 (1997).

[12] J.T. Seeman, F.-J. Decker, I. Hsu, Proceedings HEACC-92, 879 (1992).

[13] V. Shiltsev, Proceedings IWAA-95, 352 (1995).

[14] A. Seryi, "Ground Motion Models for Future Linear Colliders," Proceedings EPAC-2000 (2000).

[15] L. Hendrickson et al, Proceedings ICALEPCS-91 414 (1991).

[16] L. Hendrickson et al, Proceedings PAC-99, 338 (1999).

[17] A. Seryi, "Recent Ground Motion Studies at SLAC," Proceedings EPAC-2000 (2000).

[18] P. Tenenbaum, Proceedings PAC-99, 3459 (1999).

[19] C. Adolphsen et al, A Zeroth-Order Design Report for the Next Linear Collider (1996).

[20] B. Autin, Y. Marti, CERN-ISR-MA-73-17 (1973). 